\title{
PATRONES DE MOVILIDAD EN ÁREAS DISTANTES DE REGIONES METROPOLITANAS MULTICÉNTRICAS: RADIALIDAD VS. TANGENCIALIDAD. EL CASO DE CASTILLA-LA MANCHA RESPECTO A LA REGIÓN METROPOLITANA MADRILEÑA ${ }^{1}$
}

\author{
Inmaculada Mohíno Sanz \\ José María de Ureña Francés \\ Eloy Solís Trapero \\ Departamento de Ingeniería Civil. Escuela de Arquitectura. Universidad de Castilla-La Mancha \\ inmaculada.mohino@uclm.es ; josemaria.urena@uclm.es ; eloy.solis@uclm.es
}

\section{RESUMEN}

El artículo aborda la evolución de los patrones de movilidad laboral (commuting y negocios) de áreas distantes de Regiones Metropolitanas Multicéntricas. Tomando Madrid y Castilla La Mancha como caso de estudio, se pretende analizar la evolución (1981-2012) de las relaciones funcionales de la región castellano-manchega con la metrópoli y las tangenciales dentro de ella, con especial atención a las dirigidas a la capital regional. Los resultados muestran que frente a la predominancia de los flujos intraprovinciales y radiales, los tangenciales comienzan a adquirir importancia en los patrones de movilidad.

Palabras clave: Patrones de movilidad, Commuting, Relaciones de negocio, Regiones Metropolitanas Multicéntricas, Capital Regional.

Fecha de recepción: octubre 2013.

Fecha de aceptación: julio 2014.

1 Este trabajo es resultado de los proyectos de investigación: «La articulación de las ciudades de Castilla-La Mancha y los procesos de metropolización de Madrid facilitados por el AVE» (PPII10-0048-643) financiado por la Junta de Comunidades de Castilla-La Mancha (Investigador Principal: José María de Ureña) y «Las nuevas estructuras urbanas y territoriales de la Alta Velocidad ferroviaria en España: caracterización de la oferta y análisis de su papel en las relaciones territoriales» (TRA2011-28465-C03-01) financiado por el Ministerio de Educación (Investigador Principal: José María Coronado) así como del programa nacional de Formación de Profesorado Universitario (Convocatoria 2012). 


\begin{abstract}
Households' functional relationships in distant areas of multicentric metropolitan regions: radial versus tangential mobility patterns.

This paper aims in analyzing work-related (commuting and business) mobility patterns evolution of distant areas of Multicentric Metropolitan Regions. By focusing on the Madrid and Castilla La Mancha regions, household' functional relationships changes (1981-2012) are analysed: radial with the metropolis and tangential within the Castilla La Mancha region, paying spatial attention to those ones toward its regional capital. The empirical results show that despite de relevance of intraprovincial and radial flows, tangential linkages start acquiring importance in the mobility patterns.
\end{abstract}

Keywords: Mobility patterns, Commuting, Business travel, Multicentric Metropolitan Regions, Regional Capital cities.

\title{
I. INTRODUCCIÓN
}

Las redes de transporte han influido fuertemente en el desarrollo económico, las estructuras urbanas y los patrones de movilidad y viceversa (Biehl, 1991; Gutiérrez-Puebla y García-Palomares, 2007) y las mejoras de las últimas décadas, junto a un aumento de la movilidad, han jugado un papel clave en el devenir metropolitano, dando lugar a estructuras urbanas de mayor complejidad, donde los tradicionales modelos monocéntricos han comenzado a evolucionar hacia otros de tipo policéntrico, así como a un cambio de escala de estas regiones metropolitanas, incrementándose la separación entre orígenes y destinos (Findlay et al., 2001) y por consiguiente las distancias de viaje por motivos laborales (Rouwendal y Rietveld, 1994) y discrecionales (Schwanen et al., 2001).

En estas emergentes Regiones Metropolitanas Multicéntricas (en adelante, RMM), la distancia a la metrópoli ha dejado de constituir el principal y único criterio de localización de hogares y centros de trabajo (Filion et al, 1999), cobrando mayor relevancia la proximidad a otros subcentros. Esta menor capacidad de polarización de la metrópoli en la atracción de flujos se traduce en un cambio en los patrones de movilidad, que si bien inicialmente se trataba de unas débiles relaciones fundamentalmente radiales (hacia la metrópoli) y desde localizaciones/sub-centros próximos al centro, se han complejizado, caracterizándose no solo por dichas relaciones centrípetas o de periferia-centro (que se han reforzado en intensidad y en área de atracción) sino además centrífugas (Giuliano y Gillespie, 1997) y tangenciales o de periferia-periferia (Clark y Kuijpers-Linde, 1994; Van der Laan, 1998). Sin embargo, interesa saber hasta qué punto estas nuevas relaciones funcionales más complejas y que han comenzado a caracterizar los territorios de las tradicionales áreas metropolitanas tienen lugar en áreas más alejadas de la metrópoli.

El trabajo pretende abordar cómo la influencia metropolitana y los procesos de re-escalamiento del Estado Nación, con la consiguiente creación de las diferentes comunidades autónomas y la emergencia de nuevas centralidades regionales como es el caso de las capitales de dichas autonomías, influyen en las dinámicas de movilidad de territorios distantes de RMM 
(aproximadamente entre 50-250 km desde la metrópolis). En este sentido la hipótesis de partida sostiene que con la descentralización política y económica en capitales regionales y provinciales, éstas empiezan a adquirir una mayor capacidad de polarización, teniendo lugar una redistribución de los flujos.

Para contrastar dicha hipótesis, se propone la Región Metropolitana Madrileña como caso de estudio, como buen ejemplo de la complejidad de los emergentes sistemas urbanos debido a los cambios que han acontecido durante las últimas décadas. Por un lado, procesos de dispersión, desconcentración y relocalización de población y actividades (Solís et al., 2012) apareciendo o reforzando nuevos subcentros metropolitanos que empiezan a adquirir un papel relevante en la organización de la región. Por otro, cambios en la movilidad (García-Palomares, 2010), asociados a un fuerte desarrollo de las infraestructuras de transporte, donde los tradicionales flujos principalmente atraídos por la metrópoli han evolucionado hacia patrones más complejos y dispersos entre subcentros metropolitanos (Gallo et al., 2010).

El artículo se organiza en cuatro secciones dedicadas a la recopilación de estudios previos centrados en la compleja relación entre estructuras urbanas y relaciones funcionales, la presentación del caso de estudio, las fuentes de información y la metodología, la discusión de los resultados empíricos y las conclusiones.

\section{LOS NUEVOS PATRONES URBANOS Y DE MOVILIDAD}

Diversos académicos se han centrado en los cambios en las distancias o tiempos de viaje como consecuencia de los nuevos patrones urbanos policéntricos/dispersos (Cervero y Wu, 1998; Giuliano y Small, 1993; Gordon et al., 1991; Levinson, 1998) aunque sus conclusiones son contradictorias. Por otro lado, los estudios más recientes coinciden en que los emergentes modelos policéntricos han dado lugar a un aumento del commuting y de la dependencia del vehículo privado (Frost et al., 1998; García-Palomares, 2010; Ma y Banister, 2006) y que la «relocalización» cerca de los centros de empleo solo ocurre en determinados casos/para determinados grupos de población (Aguiléra, 2005). Por el contrario, atendiendo a diferentes motivos de viaje, Schwanen et al. (2001) concluyen que las distancias de viaje aumentan en modelos monocéntricos pero no necesariamente en los policéntricos. No obstante, la reorganización de los patrones de movilidad depende del tipo de subcentro, su tamaño y la proximidad a la metrópoli (Aguiléra y Mignot, 2004).

Esta compleja relación entre las relaciones funcionales y los patrones urbanos ha sido tema central en numerosos estudios, fundamentalmente analizada a través de flujos laborales/commuting (Castañer et al., 2000; Cervero, 1996; Clark y Kuijpers-Linde, 1994; Feria y Albertos, 2010; Reques et al., 2012; Sick y Harder, 2008), y tan solo un número reducido considera otros tipos de flujos menos recurrentes (Faulconbridge et al., 2009; Limtanakool et al., 2007a; Schwanen et al., 2001) puesto que tan solo representan una pequeña parte de la movilidad cotidiana (Nelson y Niles, 2000). En cualquier caso, puesto que el creciente aumento de la movilidad se debe en buena medida a un mayor número de desplazamientos no laborales (Ureña y Muruzábal, 2006) y debido a que los centros de trabajo han dejado de estar tan centralizados en un número reducido de centros, es creciente la relevancia de desplazamientos no obligados en los estudios de movilidad (Aguilera et al., 2009). 
Entre dichos estudios centrados en el debate entre forma urbana y movilidad, pueden encontrarse fundamentalmente dos tipos de aproximaciones.

- Por un lado, aquellos cuyo objetivo es entender el esquema de asentamientos a partir del análisis de flujos (intensidad y dirección) para diferentes motivos de viaje (Burger et al., 2011; Limtanakool et al., 2007a; 2009). De entre ellos es de especial interés el análisis que Hall y Pain (2006) realizan de las relaciones funcionales de ocho «MegaCity Regions» europeas, prestando atención no solo a las ligazones entre ciudades para cada una de ellas sino además a la importancia relativa de estas ciudades en cada una de las regiones (en función de su conectividad a escala intra-regional y global). Sin embargo, se trata de un análisis estático. Otros dos estudios de movilidad similares diferenciando los viajes polarizados hacia la metrópoli, los existentes entre regiones periféricas o distantes así como los atraídos por estas han sido llevados a cabo para la cuenca parisina ${ }^{2}$ (Gilli, 2005³) y el sureste de Inglaterra (de Goei et al., 2010 $)$.

- Por otro, aquellos cuyo debate se centra en responder si los patrones de commuting se deben a la estructura urbana. En esta línea, Giuliano y Small (1993) concluyen que otros factores deben influir además del sistema de asentamientos puesto que los tiempos de viaje son superiores a los mínimos. Es por ello que estudios posteriores se han centrado en analizar el impacto de los desarrollos urbanísticos a una escala más local (Cervero y Kockelman, 1997; Boarnet y Sarmiento, 1998; Cervero 2002; Le Néchet, 2012) sobre los comportamientos de viaje, así como la influencia de otros aspectos socioeconómicos de la población (Titheridge y Hall, 2006; Van de Coevering y Schwanen, 2006) o la estructura de los hogares (Giuliano y Gillespie, 1997; McQuaid, 2009). Por su parte, con el fin de analizar las características de los nodos y su influencia sobre los patrones de movilidad, Limtanakool et al. (2007b) centran su investigación en analizar la posición de las ciudades dentro del sistema urbano atendiendo a un análisis de flujos (por motivos de negocio y de ocio) y de los atributos de los nodos (en relación a sus características socio-demográficas, accesibilidad, economía y turismo).

El presente artículo pretende completar los diversos estudios existentes sobre los emergentes modelos de movilidad asociados a las nuevas estructuras territoriales metropolitanas con un análisis diacrónico de las ligazones laborales (tanto frecuentes como por motivos de negocio) para territorios distantes de Regiones Metropolitanas Multicéntricas (entre 50 y 250 $\mathrm{km}$ aprox.), discerniendo entre los diferentes tipos de relaciones en función de su dirección, haciendo especial énfasis a las diferencias entre los flujos jerárquicos dirigidos a la metrópoli o a otros municipios regionales de alta centralidad (capitales regionales y provinciales). La originalidad de la temática radica en tratar de entender el rol de las capitales regionales de dichos territorios alejados recientemente transformados en regiones político-administrativas frente a la tradicional polarización de la metrópoli en la atracción de flujos. En definitiva,

2 Espacio socio-económico bajo la influencia de Paris.

3 El análisis no considera otros motivos de viaje y diferentes escenarios temporales.

4 El análisis considera la evolución 1981-2001. 
el debate se centra en analizar hasta qué punto el centro metropolitano continúa liderando los patrones de movilidad en dichos territorios distantes o si por el contrario, nuevos flujos tangenciales comienzan a cobrar importancia.

\section{CASO DE ESTUDIO, FUENTES DE INFORMACIÓN Y METODOLOGÍA}

\section{III.1. El territorio castellanomanchego como caso de estudio}

Los cambios en la estructura urbana en la Región Centro española han sido notables. Desde la década de los años 80, debido a la significativa inversión en el sistema de transportes y a los procesos de descentralización de la población y las actividades económicas, nuevos centros económicos y de empleo han comenzado a emerger en torno a Madrid, consolidándose una RMM (Solís, 2012). Como consecuencia, los procesos metropolitanos han comenzado a desbordar más allá de los propios límites administrativos de la provincia/región madrileña, dando lugar a fuertes interrelaciones funcionales con las provincias adyacentes (Burns et al., 2009; García-Palomares, 2010; Méndez y Rodríguez, 2007; Valenzuela, 2011).

Con el fin de analizar los patrones de movilidad y las emergentes centralidades en territorios distantes de RMM, el área de estudio (Fig.1 y Tabla 1) abarca las comunidades autónomas de Madrid y Castilla-La Mancha (en adelante, CLM). Consolidada en 1982 como región político-administrativa con Toledo como capital regional, CLM ha sido tradicionalmente considerada como un territorio desorganizado, sin cohesión funcional y acefálico, notablemente influido por Madrid (Cebrián y Cebrián, 2000).

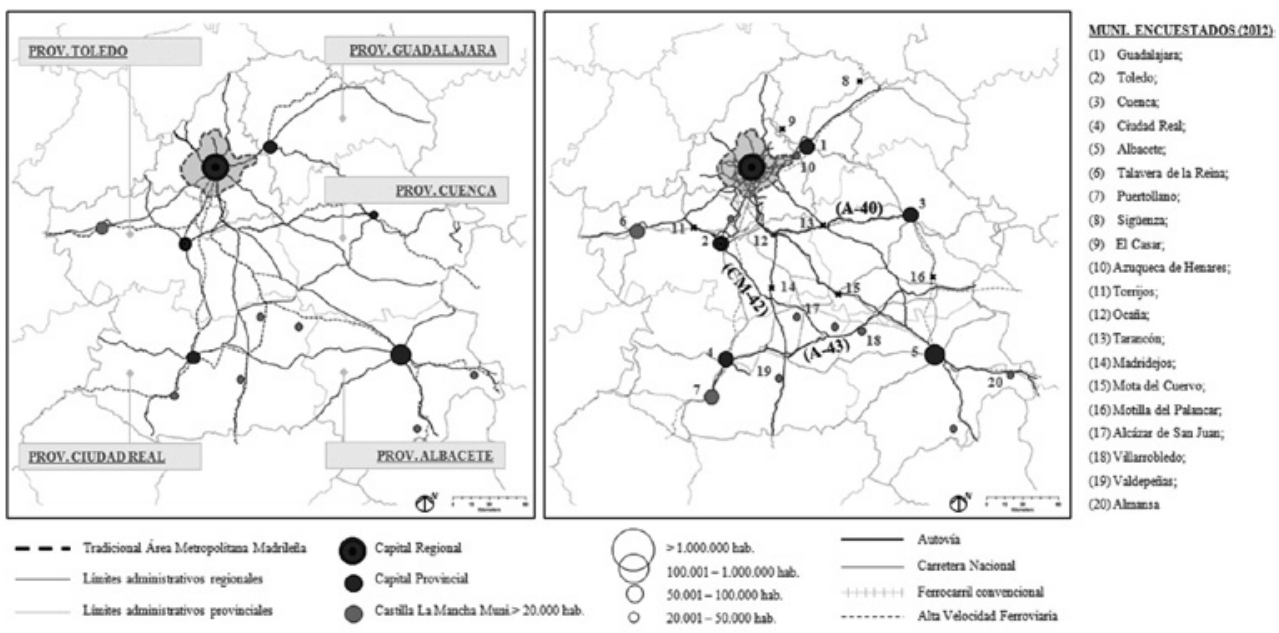

Fuente: elaboración propia. Información tomada del Centro Nacional de Información Geográfica - CNIG, Censo de Población y Viviendas de 1981 - INE- y Padrón municipal 2012 - INE). 
Tabla 1

EVOLUCIÓN DE LA POBLACIÓN DE LAS PROVINCIAS Y CAPITALES DE CASTILLA LA MANCHA (1981-2012)

\begin{tabular}{|c|c|c|c|c|c|c|}
\hline & \multicolumn{2}{|c|}{ Población (1981) } & \multicolumn{2}{|c|}{ Población (2001) } & \multicolumn{2}{|c|}{ Población (2012) } \\
\hline & (Hab.) & $(\%)$ & (Hab.) & $(\%)$ & (Hab.) & $(\%)$ \\
\hline Albacete (Capital) & 117.126 & 35 & 149.507 & 41 & 172.472 & 43 \\
\hline Otros municipios $>20.000$ hab. $\left(\mathrm{N}^{\circ}\right)$ & $42.982(2)$ & 13 & $74.327(3)$ & 20 & $83.219(3)$ & 21 \\
\hline Resto de la provincia de Albacete & 174.360 & 52 & 143.449 & 39 & 147.146 & 37 \\
\hline Total Provincia Albacete & 334.468 & 100 & 367.283 & 100 & 402.837 & 100 \\
\hline Ciudad Real (Capital) & 51.118 & 11 & 61.280 & 13 & 74.921 & 14 \\
\hline Otros municipios $>20.000$ hab. $\left(\mathrm{N}^{\circ}\right)$ & $125.533(4)$ & 27 & $132.342(4)$ & 28 & $154.294(4)$ & 29 \\
\hline Resto de la provincia de Ciudad Real & 291.676 & 62 & 284.959 & 60 & 301.035 & 57 \\
\hline Total Provincia Ciudad Real & 468.327 & 100 & 478.581 & 100 & 530.250 & 100 \\
\hline Cuenca (Capital) & 41.791 & 20 & 46.491 & 23 & 57.032 & 26 \\
\hline Otros municipios $>20.000$ hab. $\left(\mathrm{N}^{\circ}\right)$ & 0 & 0 & 0 & 0 & 0 & 0 \\
\hline Resto de la provincia de Cuenca & 167.207 & 80 & 155.035 & 77 & 161.004 & 74 \\
\hline Total Provincia Cuenca & 208.998 & 100 & 201.526 & 100 & 218.036 & 100 \\
\hline Guadalajara (Capital) & 56.922 & 54 & 67.640 & 39 & 84.803 & 33 \\
\hline Otros municipios $>20.000$ hab. $\left(\mathrm{N}^{\circ}\right)$ & 0 & 0 & $20.383(1)$ & 12 & $35.146(1)$ & 14 \\
\hline Resto de la provincia de Guadalajara & 48.097 & 46 & 83.509 & 49 & 139.588 & 54 \\
\hline Total Provincia Guadalajara & 105.019 & 100 & 171.532 & 100 & 259.537 & 100 \\
\hline Toledo (Capital) & 57.769 & 12 & 69.450 & 13 & 84.019 & 12 \\
\hline Otros municipios $>20.000$ hab. $\left(\mathrm{N}^{\circ}\right)$ & $64.136(1)$ & 14 & $76.011(2)$ & 14 & $113.336(2)$ & 16 \\
\hline Resto de la provincia de Toledo & 349.901 & 74 & 390.670 & 73 & 513.873 & 72 \\
\hline Total Provincia Toledo & 471.806 & 100 & 536.131 & 100 & 711.228 & 100 \\
\hline Total Región Castilla La Mancha & 1.588 .618 & & 1.755 .053 & & 2.121 .888 & \\
\hline
\end{tabular}

Fuente: Censos de Población y Viviendas de 1981 y 2001 y Padrón municipal de 2012 (INE).

El carácter de paso del sistema de transporte de CLM ha sido un condicionante clave en la dinámica urbana regional y a pesar de las mejoras de transporte de las últimas décadas, que han comenzado a transformar la tradicional red de ferrocarril y carreteras, predominantemente radial, en otra radioconconcéntrica en torno a la metrópoli, en el territorio castellanomanchego las infraestructuras continúan siendo proyectadas bajo lógicas nacionales conectando la región con Madrid. En cuanto a la red de carreteras, los principales cambios que han tenido lugar en el área de estudio han sido principalmente tres: a) la adecuación (o duplicación) de las carreteras nacionales a los estándares de autovía en todo el área de estudio, reduciendo considerablemente los tiempos de viaje (ver Tabla 2); b) la construcción de cuatro nuevas autovías orbitales/semi-orbitales en la provincia/región madrileña (M-30, M-40, M-45 y M-50); c) construcción de tres tramos de autovía tangenciales (CM42, A-43 y A-40) en CLM. En cuanto a la red ferroviaria, los cambios han consistido en la reducción del número de servicios de la red convencional, la ampliación del ferrocarril 
suburbano/cercanías más allá de los límites de la región madrileña y la creación de la red de Alta Velocidad entre cada una de las provincias de CLM y Madrid5.

Tabla 2

EVOLUCIÓN DE LOS TIEMPOS DE VIAJE POR CARRETERA ENTRE LAS PRINCIPALES CIUDADES DE MADRID Y CASTILLA LA MANCHA. (AÑOS 1981-2012)

\begin{tabular}{|c|c|c|c|c|c|c|c|c|c|c|}
\hline \multicolumn{2}{|c|}{ Ciudad Real } & \multicolumn{2}{|c|}{ Cuenca } & \multicolumn{2}{|c|}{ Guadalajara } & \multicolumn{2}{|c|}{ Madrid } & \multicolumn{2}{|c|}{ Toledo } & \\
\hline $\begin{array}{c}1981 \\
(\mathrm{~min})\end{array}$ & $\begin{array}{c}2012 \\
(\% \\
\text { reduct })\end{array}$ & $\begin{array}{l}1981 \\
(\min )\end{array}$ & $\begin{array}{c}2012 \\
(\% \\
\text { reduct })\end{array}$ & $\begin{array}{l}1981 \\
(\min )\end{array}$ & $\begin{array}{c}2012 \\
(\% \\
\text { reduct })\end{array}$ & $\begin{array}{c}1981 \\
(\mathrm{~min})\end{array}$ & $\begin{array}{c}2012 \\
(\% \\
\text { reduct })\end{array}$ & $\begin{array}{c}1981 \\
(\mathrm{~min})\end{array}$ & $\begin{array}{c}2012 \\
(\% \\
\text { reduct })\end{array}$ & \\
\hline \multirow[t]{5}{*}{134} & 19 & 112 & 25 & 192 & 31 & 151 & 22 & 156 & 20 & Albacete \\
\hline & & 145 & 31 & 159 & 16 & 132 & 11 & 80 & 0 & Ciudad Real \\
\hline & & & & 83 & 0 & 99 & 22 & 117 & 26 & Cuenca \\
\hline & & & & & & 34 & 27 & 80 & 26 & Guadalajara \\
\hline & & & & & & & & 47 & 23 & Madrid \\
\hline
\end{tabular}

Fuente: elaboración propia.

La mayor parte de los estudios de movilidad en la Región Centro española han limitado sus análisis a territorios cercanos a la metrópoli, aproximadamente a unos $50 \mathrm{~km}$ en torno a ella (Gallo et al., 2010; García-Palomares, 2010; Gutiérrez-Puebla y García-Palomares, 2007) y tan solo una minoría considera áreas más alejadas (Pillet et al., 2010; Solís et al., 2012). Por otro lado, debido a la falta de datos de movilidad actualizados/recientes, principalmente se han limitado a analizar la evolución de ligazones laborales para un período de veinte años (1981-2001). Por ello, el presente artículo pretende ir más allá, tratando de analizar la evolución hasta la situación actual (1981-2012) no solo de los viajes de commuting sino de un tipo de viaje que comienza a cobrar especial importancia en los patrones de movilidad obligada, los viajes de negocio, y cubriendo un territorio más distante del hasta ahora considerado en este tipo de estudios.

\section{III.2. Metodología}

El presente análisis de articulación territorial se aborda desde una doble perspectiva.

- Por un lado, atendiendo a la evolución de los patrones de commuting ocurridos en las tres últimas décadas (1981-2001-2012). En primer lugar, en términos absolutos (número de viajes originados en cada municipio) con el fin de identificar aquellos centros del sistema de ciudades liderando los patrones de movilidad regional (desde el punto de vista de la intensidad de la interacción). En segundo lugar, en términos relativos (como el porcentaje de desplazamientos intermunicipales que desde un municipio se dirigen a un determinado destino) con el fin de entender los cambios de centralidad de los diferentes destinos.

5 Existen tres conexiones tangenciales regionales de alta velocidad ferroviaria: CR-Cuenca (un único servicio por día y dirección), Cuenca-Albacete y CR-Puertollano (no relevante desde el punto de vista de relaciones horizontales debido a la reciente autovía que conecta igualmente ambos municipios). 
- Por otro, caracterizando los patrones regionales de desplazamientos por motivos de negocio, comparando su estructura con la de commuting y analizando la evolución de dichas relaciones de negocio (2006-2012) para la provincia de Ciudad Real.

Para cada uno de los municipios, se prestará especial atención a las relaciones intermunicipales determinantes, definidas como aquellas con más de 50 ligazones y en las que los desplazamientos intermunicipales representan más del $10 \%$ de la población ocupada desplazada. De entre ellas, se distinguirá igualmente entre la relación con el mayor número de desplazamientos (primaria) del resto (secundarias).

Con el fin de caracterizar la capacidad de polarización de las principales centralidades de RMM, en estos análisis se discretizarán las relaciones funcionales en función del tipo de destino, distinguiendo entre:

a) Relaciones intraprovinciales jerárquicas: desplazamientos intermunicipales con destino la capital de la provincia de residencia.

b) Relaciones intraprovinciales no jerárquicas: desplazamientos intermunicipales con destino otro municipio de la provincia de residencia (excluyendo la capital).

c) Relaciones intraprovinciales totales, como suma de los dos tipos de flujo anteriores, es decir, desplazamientos intermunicipales con destino otro municipio de la provincia de residencia.

d) Relaciones tangenciales jerárquicas: desplazamientos intermunicipales con destino la capital regional (en el caso del área de estudio, Toledo).

e) Relaciones tangenciales no jerárquicas: desplazamientos intermunicipales con destino otras provincias castellanomanchegas (excepto al municipio de Toledo).

f) Relaciones tangenciales totales, como suma de los dos tipos de flujo anteriores, es decir, desplazamientos intermunicipales con destino otro municipio regional (de una provincia diferente a la de residencia).

g) Relaciones radiales jerárquicas: desplazamientos intermunicipales con destino el municipio de Madrid.

h) Relaciones radiales no jerárquicas: desplazamientos intermunicipales con destino con otros municipios madrileños (excluyendo Madrid).

i) Relaciones radiales totales, como suma de los dos tipos de flujo anteriores, es decir, desplazamientos intermunicipales con destino otro municipio de la provincia madrileña.

j) Relaciones inter-regionales: desplazamientos intermunicipales hacia otras regiones españolas, excepto la madrileña.

k) Relaciones internacionales: desplazamientos intermunicipales hacia otros países.

\section{III.3. Fuentes de información}

Las estadísticas del Instituto Nacional de Estadística (INE), y en concreto los Censos de Población y Vivienda de 1981 y 2001 y su información referente a la movilidad labo$\mathrm{ral} /$ desplazamientos residencia-trabajo, conforman una de las principales fuentes de datos empleadas. A pesar de que el Censo de 1981 incluía en su cuestionario una pregunta referente al municipio de trabajo/estudio, la información recogida presenta algunos problemas 
o carencias, como el ocurrido a la hora de llevar a cabo su explotación (tan solo se dispone de información desagregada de los desplazamientos hacia municipios de más de 20.000 habitantes), lo cual ha limitado de manera significativa su utilización. El Censo de 2001, es el más novedoso y fructífero de la historia censal del país, ya que introduce un gran número de respuestas y un cruce de numerosas variables con la posibilidad de obtener un perfil de la población más riguroso. Este Censo recoge en su cuestionario una pregunta referente al lugar de trabajo de la población mayor de 16 años, diferenciando cinco categorías: aquellos que trabajan/estudian en el propio domicilio, en el mismo municipio, en varios municipios (transportistas, representantes, etc), en otro municipio (identificando cuál) o en otro país. Esta fuente introduce el concepto de población vinculada que es definida como el conjunto de personas censales (residentes en España) que tienen algún tipo de vinculación con el municipio en cuestión (ya sea por residencia, trabajo o estudios).

Sin embargo, uno de los retos de la presente investigación es la falta de información estadística pública accesible y actualizada a escala supra-metropolitana para el caso de desplazamientos obligados de commuting (no disponible en el área de estudio desde 2001). En cuanto a los viajes por motivos de negocio, dicha información tan solo se encuentra disponible para una de las provincias (Ciudad Real) y para los escenarios de 1980 y 2006, a través de las encuestas realizadas por las investigaciones de Pillet et al. (1980) y Garmendia et al. (2011). Por ello, surge la necesidad de llevar a cabo una encuesta de movilidad de los hogares regionales, diseñada, realizada y explotada por los propios autores, con el fin entender los patrones de desplazamientos en 2012.

Por tratarse de un territorio muy extenso $\left(79.463 \mathrm{~km}^{2}\right)$ y alta población (2.121.888 hab en 2012), la realización de encuestas con una distribución homogénea, fue descartada desde un primer momento, debido a la imposibilidad técnica en términos de recursos humanos y económicos necesarios para llevar a cabo la recogida y el tratamiento de la información, así como de su duración. La metodología empleada a la hora de escoger la muestra consistió en dirigir los cuestionarios a un sector específico de la población siguiendo una metodología probada con anterioridad y cuyos resultados permitieron establecer conclusiones relevantes en los patrones de movilidad (Pillet, 1980; Garmendia et al., 2011). Se trata de «organizar una estructura de distribución y recogida de los cuestionarios en forma de árbol» (Pazos, 2005) con una serie de personas clave de contacto directo, que a su vez harían llegar las encuestas al total de la muestra. Se seleccionó distribuir los cuestionarios entre los alumnos de Educación Secundaria Obligatoria y Bachillerato. A pesar del sesgo producido al considerar únicamente un determinado grupo de población (familias con hijos en edad escolar), este sector presenta altos niveles de movilidad (Schwanen et al., 2001) haciéndolo más atractivo para el estudio (Pazos, 2005).

Una vez definida la metodología, los municipios a los que dirigir el cuestionario (un total de 20) fueron seleccionados (ver Figura 1) según los siguientes criterios:

1. Su localización geográfica con el fin de alcanzar un conjunto de puntos de contacto homogéneamente distribuidos por el territorio regional;

2. Su tamaño en términos de población y la existencia de, al menos, un instituto de Educación Secundaria Obligatoria;

3. Una dinámica demográfica positiva durante los últimos treinta años (1981-2012) y

4. Su capacidad de articular/organizar los territorios adyacentes 
El tamaño de la muestra se calculó para un margen de error del $7 \%$ y un nivel de confianza del $95 \%$. Un total de 7345 encuestas fueron completadas y recibidas, de las cuales se rescataron 6913 encuestas válidas, cumpliendo con los objetivos de representatividad de la muestra fijados.

El cuestionario se elabora en torno a dos bloques: uno primero más general, enfocado a caracterizar el hogar y en concreto, al cabeza de familia encuestado, y un segundo que tiene un carácter más detallado y cuya finalidad es obtener información de los desplazamientos. En este segundo bloque se distinguen además dos partes. Una primera destinada a los desplazamientos del cabeza de familia (tanto por motivos de trabajo como por relaciones de negocio con municipios diferentes al de residencia) y por otro, del resto de miembros de la unidad familiar que se desplazan a diario al centro de trabajo a otro municipio diferente al de residencia. La segunda parte del bloque hace referencia al resto de posibles desplazamientos en función de distintos motivos de viaje (compras, sanitario, ocio, etc.). Los análisis presentados en este artículo se centran únicamente en la información recogida en el primer bloque, esto es, en los desplazamientos por motivos laborales: de commuting (entendidos como los desplazamientos frecuentes entre el lugar de residencia y el lugar de trabajo) y de negocio (entendidos como aquellos viajes que por motivos laborales tienen un carácter menos recurrente que los de commuting, como por ejemplo para visitar a un cliente, participar en una conferencia o asistir a una reunión).

\section{RESULTADOS EMPÍRICOS: HACIA LA MULTIDIRECCIONALIDAD DE LOS FLUJOS LABORALES}

Tal y como se ha mencionado anteriormente, el objetivo del presente estudio es, centrándose en las ligazones laborales, tratar de esclarecer si la complejidad de los emergentes modelos urbanos (policéntricos) así como de los patrones de movilidad en entornos cercanos de áreas metropolitanas, también caracteriza territorios más distantes de RMM. Para ello no solo serán significativos los flujos diarios por motivos de trabajo (commuting) sino además las relaciones de negocios, las cuales comienzan a ser cada vez más frecuentes (Urry, 2003). El interés en prestar atención a ambos motivos de viajes laborales radica en que los últimos pueden reorganizarse espacialmente con mayor rapidez y son más flexibles que los de commuting (Burger et al., 2011), lo cual podría ser un indicador de futuras reorganizaciones espaciales.

\section{IV.1. Notable aumento y mayor multidireccionalidad de los patrones de commuting.}

\section{IV.1.1. Incremento de la movilidad}

Los flujos de commuting intermunicipales han aumentado notablemente tanto en intensidad (número de desplazamientos en las diferentes relaciones) como en área de influencia (número de destinos) entre 1981 y 2012 (Fig.2.a. y Tabla A), multiplicándose los de 1981 entre 2 y 10 veces en 2001 y entre 3 y 20 veces en 2012 (Tabla 3). Este incremento notable, mayor que el ya descrito para el caso nacional (Castañer et al., 2000), podría ser debido a motivos generales como la duplicación tanto de los niveles de motorización ${ }^{6}$ como de la incorporación de la mujer al mercado laboral ${ }^{7}$, pero además a las considerables reducciones en los

6 De 0.28 veh/persona en 1981 a 0.67 veh/persona en 2011 (DGT, 2008 y 2011).

7 De $26.8 \%$ en 1981 (Martín, 2000) a 52.9\% en 2002 (INE, 2011). 


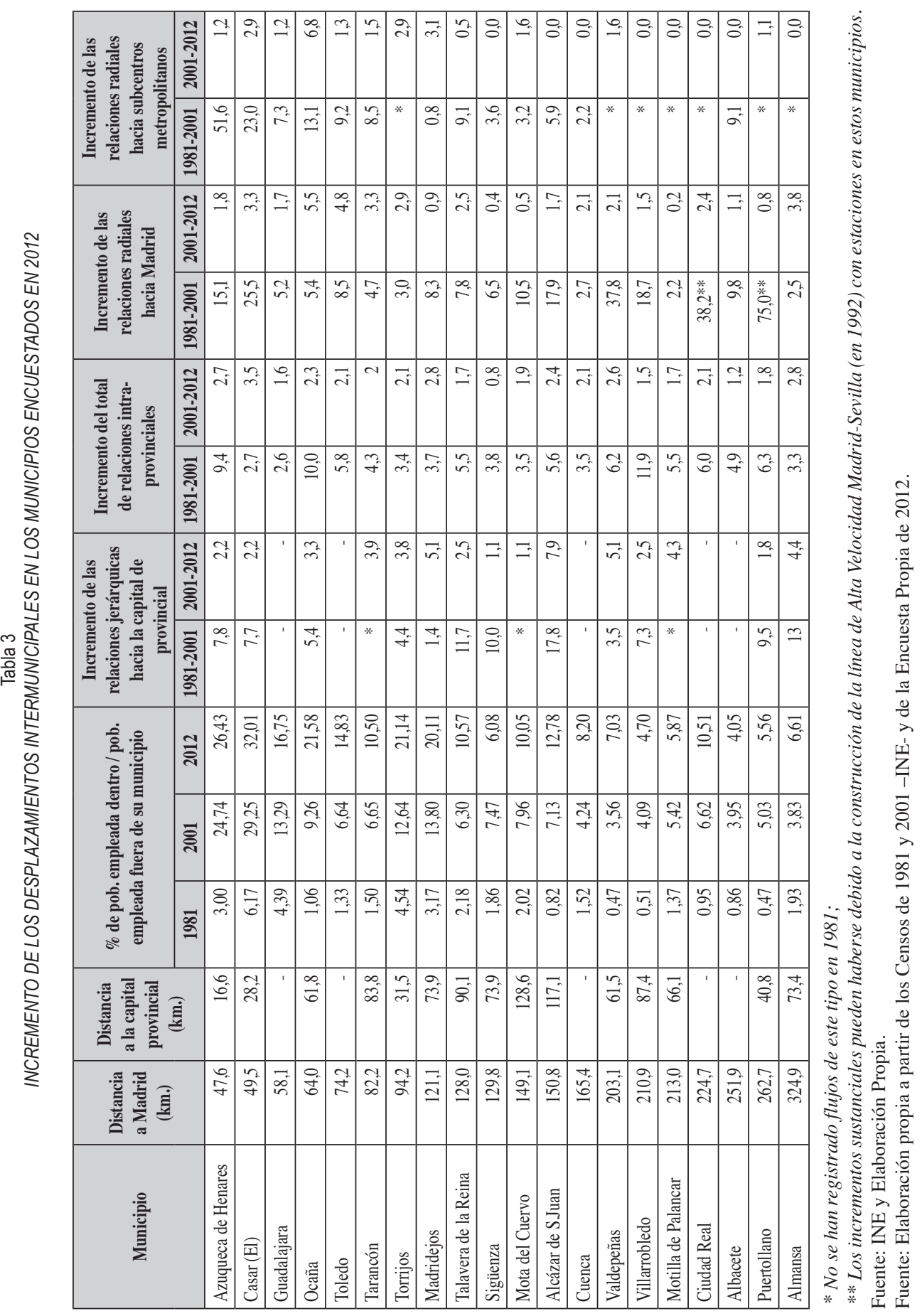


tiempos de viaje favorecidas por la mejora de las infraestructuras (en torno a un 20\%, Tabla 2) en CLM así como a la progresiva integración de dicha región en los procesos metropolitanos.

En este sentido, entre 1981 y 2012, las relaciones de commuting intraprovinciales (por lo general mayores hacia las capitales provinciales ${ }^{8}$ ) y radiales (generalmente mayores hacia Madrid $^{9}$ ) han aumentado considerablemente (Tabla 3 y Tabla A). En el caso de las interacciones radiales, este crecimiento fue más significativo entre 1981 y 2001, momento en el que otros municipios madrileños comenzaron a adquirir un papel de subcentros metropolitanos.

Desde el punto de vista de los flujos tangenciales hacia Toledo, mientras que este tipo de relación no existía en 1981 (Fig. 2.a y Tabla A), gracias a su designación como capital regional de CLM en 1982, comienzan a aparecer en 2001, excepto para los municipios de la provincia de Guadalajara (más cercanos a Madrid). En 2012, la reducción de los tiempos de viaje como consecuencia de la construcción de la nueva autovía CM-42, ha tenido un notable impacto en las relaciones hacia Toledo, aumentando desde el área central de CLM (Alcázar de San Juan, Tarancón y Villarrobledo). En 2001 y 2012, los municipios que presentan una mayor interacción con la capital regional en términos absolutos (número de desplazamientos) son las otras cuatro capitales de provincia.

Por el contrario, en cuanto al resto de flujos tangenciales, aunque con una intensidad muy débil, ya existían en algunos municipios (doce de veinte encuestados) en 1981 (Fig. 2.a y Tabla A). En 2001, todos los municipios encuestados mostraban este tipo de relación, aumentando en 2012 para la mayoría de ellos (quince de veinte municipios) (Fig.2.a. y Tabla A). En este sentido, esta multinodalidad de los patrones de movilidad sostiene nuestra hipótesis de partida y por tanto se podría concluir que nuevas centralidades están emergiendo en la región castellanomanchega.

De manera similar, las relaciones hacia otros territorios nacionales eran débiles en 1981 (Fig.2.a. y Tabla A), más significativas en aquellos territorios más distantes de Madrid (en concreto los municipios del este, tradicionalmente más relacionados con las áreas metropolitanas del levante español). El período entre 1981 y 2001 se caracteriza por un incremento generalizado de este tipo de flujos, mientras que entre 2001 y 2012, se produce una reorganización, incrementando para las dos capitales más distantes a Madrid (Albacete y Cuenca) y decreciendo en las otras tres provincias (Fig.2.a.).

Centrándonos en los flujos determinantes para el escenario de 2012 (Fig.2.b.), que han incrementado progresivamente en las últimas tres décadas, se observa que los principales nunca han sido tangenciales hacia Toledo, siendo en la mayoría de los casos radiales hacia Madrid. Las únicas excepciones se dan en:

- Municipios a menos de $60 \mathrm{~km}$ de su capital provincial, donde los flujos jerárquicos hacia dichas capitales son los principales.

810 de 15 municipios encuestados (excluyendo las cinco capitales) entre 1981 y 2001 , y 11 de 15 entre 2001 y 2012, tienen mayores incrementos de dichas relaciones intraprovinciales hacia sus respectivas capitales provinciales que hacia el resto del territorio provincial.

9 La mayoría de los flujos radiales hacia otros subcentros metropolitanos se originan en municipios cercanos a los límites administrativos de la Comunidades Autónomas (Fig.2.a). En 2001 y 2012, 18 de los 20 municipios encuestados tuvieron una mayor interacción con Madrid que con otros subcentros metropolitanos. Solo aquellos municipios más cercanos a los límites regionales $(<15 \mathrm{~km}$ ) y a otras ciudades madrileñas (con una población superior a 50,000 habitantes) presentan mayores interacciones con subcentros metropolitanos adyacentes. 
Figura 2.a

DESPLAZAMIENTOS INTERMUNICIPALES POR MOTIVOS DE COMMUTING DESDE LOS MUNICIPIOS ENCUESTADOS $\left(1981^{*}, 2001,2012\right)$

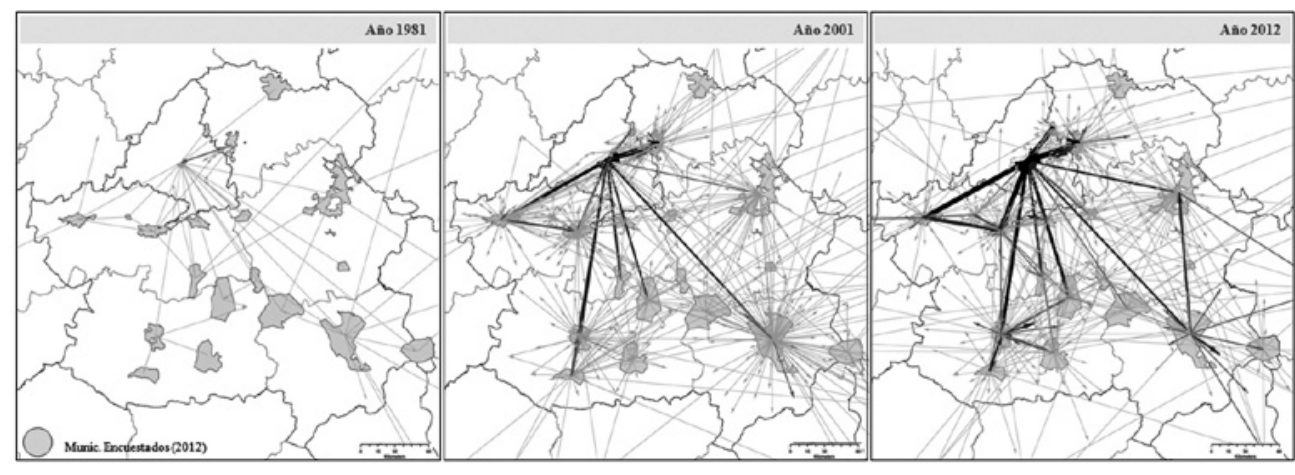

Desplazamientos intemunicipales $\left(0^{\circ}\right.$

$\longrightarrow 10.250 \longrightarrow 251.500 \longrightarrow 501 \cdot 1000 \longrightarrow 1001 \cdot 2000 \longrightarrow 2000$

Fuente: Elaboración Propia a partir de los Censos de 1981 y 2001 -INE- y de la Encuesta Propia de 2012.

Figura 2.b

DESPLAZAMIENTOS DETERMINANTES POR MOTIVOS DE COMMUTING

DESDE LOS MUNICIPIOS ENCUESTADOS $\left(1981^{*}\right.$, 2001, 2012)

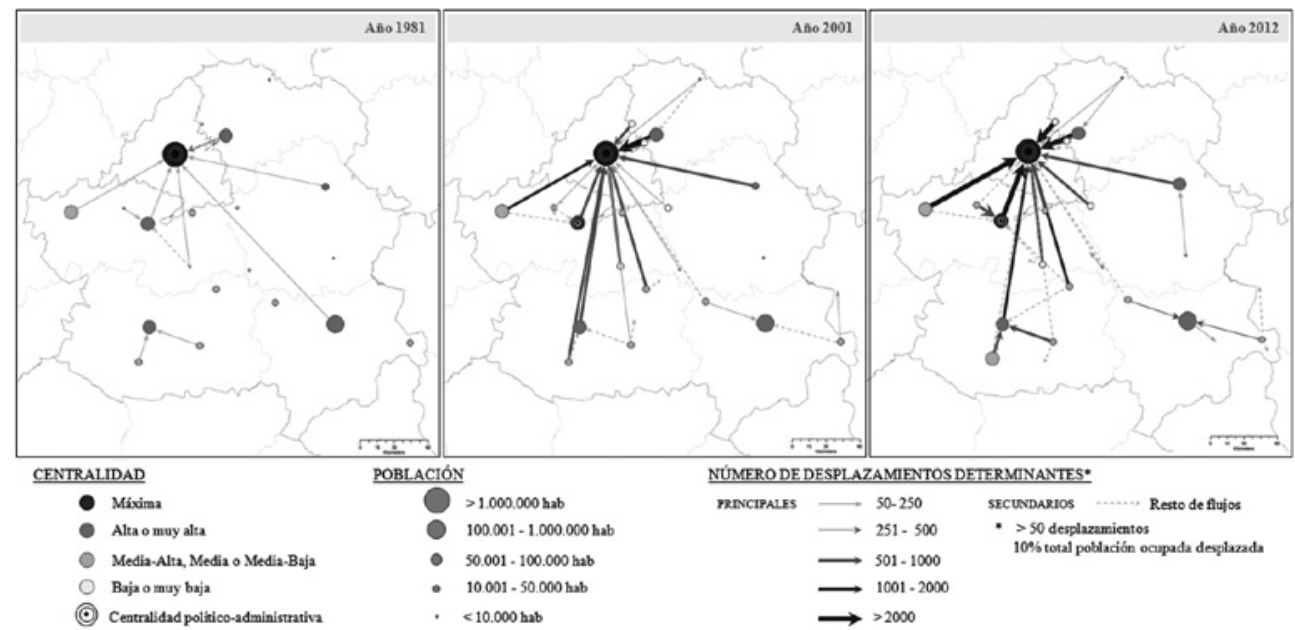

Fuente: elaboración propia a partir de los Censos de 1981 y 2001 -INE- y de la Encuesta Propia de 2012.

* La información desagregada en 1981 tan solo se encuentra disponible para los municipios de más de 20.000 habitantes. 
- Municipios de baja centralidad cercanos a los límites provinciales y a municipios de otras provincias de mayor centralidad, donde los flujos tangenciales son significativos.

- Municipios distantes de Madrid (más de 200 km) donde las relaciones intraprovinciales jerárquicas hacia la capital son las principales. Mientras que estos municipios no presentan flujos determinantes principales hacia la región madrileña, en algunos casos sí que presentan relaciones determinantes secundarias hacia Madrid u otras regiones españolas.

En cuanto a los flujos determinantes secundarios en 2012 (Fig.2.b.) en la mayoría de los casos son jerárquicos hacia la capital provincial o radiales hacia Madrid, y tan solo en dos municipios en el centro de la región son hacia otros territorios regionales. En cuanto a los flujos determinantes secundarios radiales hacia otros subcentros madrileños, únicamente tienen lugar desde municipios cercanos (aunque durante las tres últimas décadas el área de influencia comienza a cubrir un mayor número de municipios).

\section{IV.1.2. Hacia la multidireccionalidad}

Además de prestar atención a la intensidad de los flujos, es fundamental el peso relativo que cada tipo de relación representa sobre el total de las ligazones de commuting generadas desde ese municipio (Tabla 4) para entender si las centralidades que comienzan a emerger (nuevos destinos de desplazamiento) están reorganizando los patrones de movilidad.

En 1981, los flujos de commuting intermunicipales eran principalmente radiales e intraprovinciales y tan solo dos de los municipios encuestados en 2012 presentaban una notable interacción (superior al 20\%) con otros territorios regionales. Este papel fundamental ejercido por los destinos de la misma provincia de residencia ha continuado caracterizando la movilidad laboral en las siguientes décadas. Sin embargo, en 2001 las relaciones radiales comenzaron a adquirir importancia (trece municipios aumentaron su interacción con otros municipios madrileños mientras que sus relaciones tangenciales disminuyeron), perdiendo relevancia en 2012. Esta menor polarización de los municipios madrileños como principal destino de commuting refuerza nuevamente nuestra hipótesis de partida, concluyéndose que dentro del territorio castellanomanchego nuevas centralidades han comenzado a emerger y comienzan a ejercer un papel crucial en la atracción de flujos. En este sentido es importante matizar que esta menor polarización no significa un menor número de desplazamientos hacia la provincia de Madrid (ya que como se ha señalado en el apartado anterior, entre 1981 y 2012 las relaciones de commuting radiales han aumentado considerablemente en términos absolutos), sino que, debido a la creación de la capitalidad regional en Toledo que ha generado sus propios flujos de atracción, ha tenido lugar una redistribución de las ligazones laborales.

Prestando atención únicamente a los flujos hacia otras provincias diferentes a la de residencia (extra-provinciales), en 1981 podrían establecerse dos patrones. Por un lado, los municipios más cercanos a la metrópoli, donde la atracción de la región madrileña era predominante. Por otro, municipios más alejados/periféricos, que presentan una mayor vinculación hacia otras regiones españolas. En 2001, a pesar del cambio de escala en el área de atracción de la región madrileña (cubriendo un territorio más amplio/alejado) y del refuerzo 


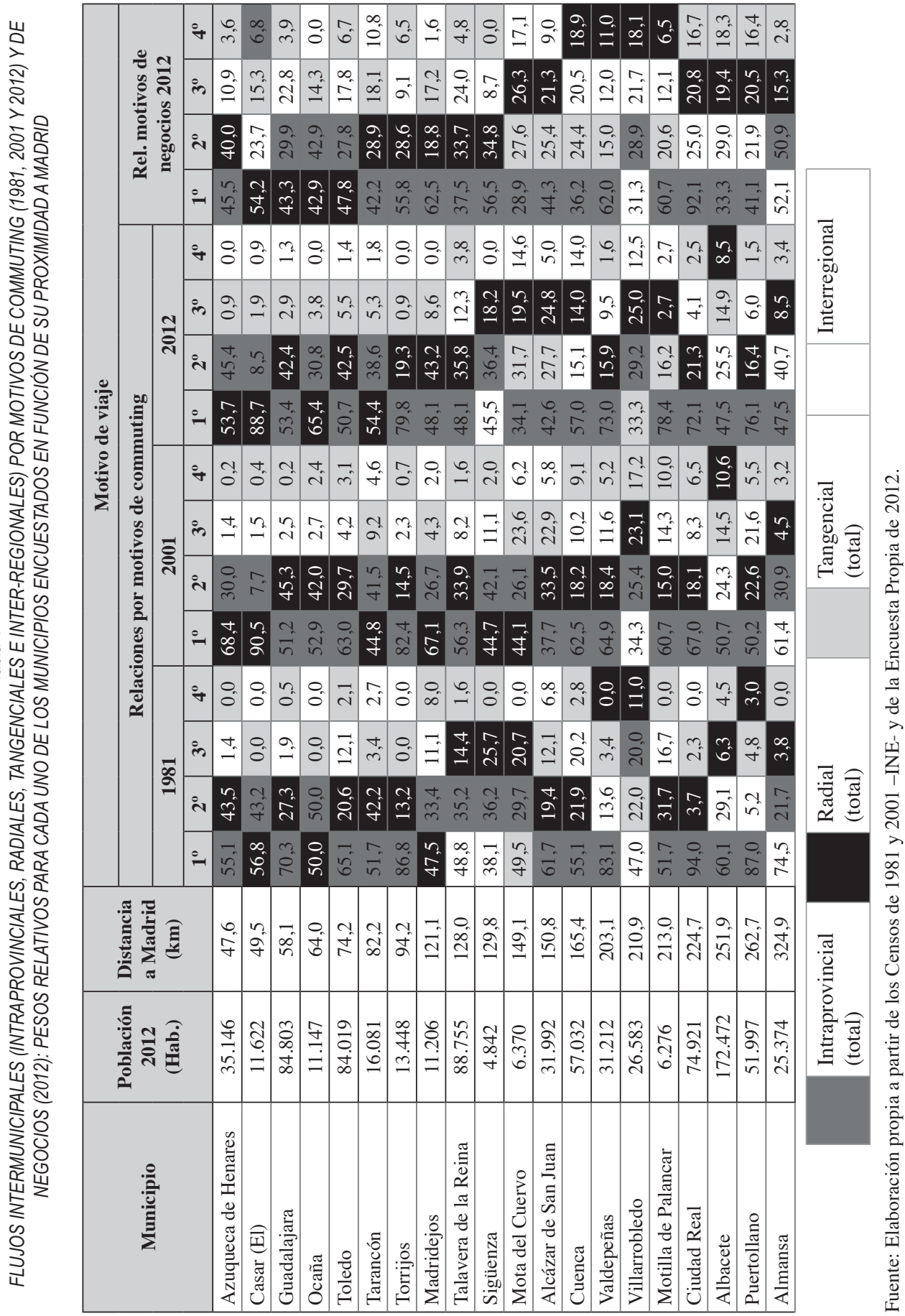


de las relaciones existentes en los municipios más cercanos a Madrid, las ligazones laborales tangenciales comienzan a ganar importancia. En 2012, el territorio donde la influencia de Madrid es sustancial/principal (frente al resto de relaciones) abarca unos $130 \mathrm{~km}$ (y tan solo en unos pocos municipios algo más alejados de la metrópoli, en cuyo caso se han visto favorecidos por la Alta Velocidad Ferroviaria), mientras que para municipios más distantes las relaciones tangenciales adquieren un papel principal sobre el resto.

En definitiva, atendiendo a la importancia relativa de las relaciones inter-municipales, se observan dos tendencias (ver Figura 3). La primera, en el período 1981-2001, donde Madrid refuerza su papel como principal atractor de flujos extraprovinciales, tanto para los municipios más cercanos como para los más alejados. La segunda, durante la última década, caracterizada por un debilitamiento del papel polarizador de Madrid junto con un refuerzo de las relaciones tangenciales (aunque todavía débiles y solo para un reducido número de municipios cercanos la zona central/centro geográfico/de gravedad de CLM), dando lugar a unos patrones de movilidad más complejos.

Figura 3

EVOLUCIÓN DE LA CAPACIDAD DE LAMETRÓPOLI DE ATRACCIÓN DE FLUJOS DE COMMUTING (1981-2012)
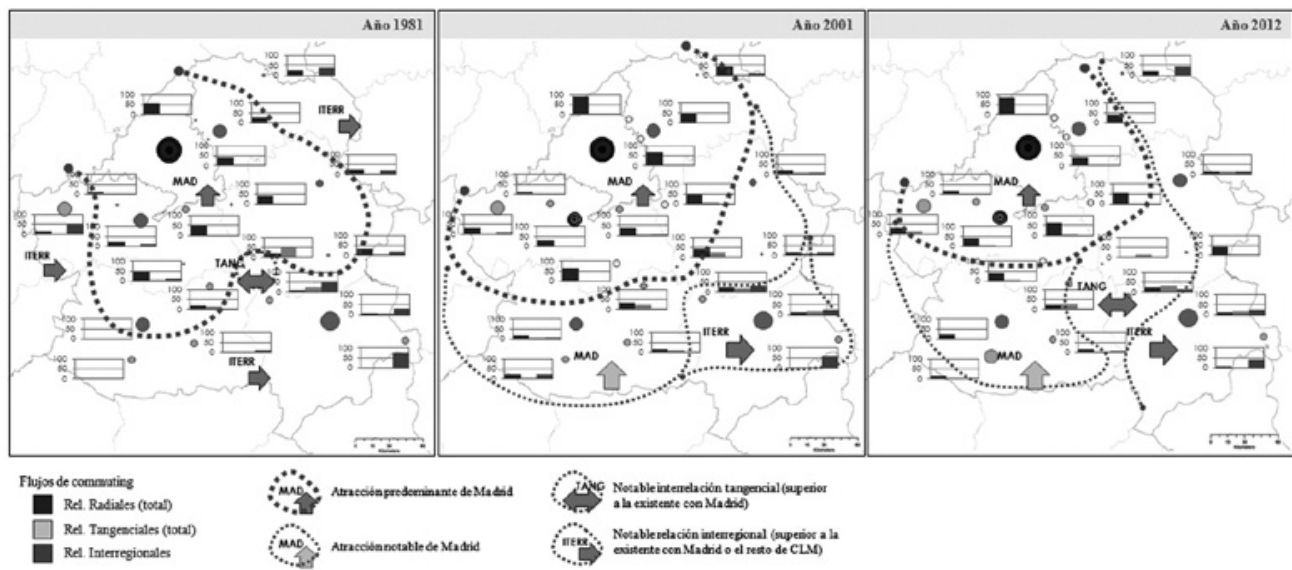

Fuente: Elaboración propia a partir de los Censos de 1981 y 2001 -INE- y de la Encuesta Propia de 2012.

\section{IV.2. Patrones espaciales de los viajes de negocios}

El análisis diacrónico de los viajes de negocios de la provincia de Ciudad Real para 2006 y 2012 revela igualmente la consolidación de unos patrones de movilidad más complejos debidos a la diversificación de los destinos. En ese sentido, mientras que en 2006 las relaciones radiales e intraprovinciales hacia cada capital $(35,0 \%$ y $21,0 \%$, respectivamente) presentaban una mayor intensidad que las tangenciales hacia Toledo $(6,0 \%)$ y las intra-regionales (Garmendia et al, 2011), en 2012, aunque los dos primeras continúan siendo principales, redujeron su atracción a la mitad $(19,0 \%$ y $8,4 \%$, respectivamente), aumentando por otro lado las ligazones tangenciales hacia Toledo en un tercio (a 9,4\%), haciéndose igualmente más significativos los flujos hacia el resto del territorio regional $(8,4 \%)$ y nacional $(19,4 \%)$. 
Por otro lado, comparando los patrones regionales de commuting y negocios para el año 2012, pueden extraerse tres conclusiones principales (Fig. $4^{10}$ ). En primer lugar, que las relaciones de negocios tienen lugar con territorios más alejados. En segundo, que las relaciones de negocios son menos complejas ya que los flujos se dirigen hacia un reducido número de destinos de alta centralidad (principalmente Madrid y en menor medida, las capitales de provincia). Y finalmente, que el total de relaciones tangenciales por motivos de negocio son claramente superiores a las que se producen por motivos de commuting (ver Tabla 4 y Figura 4).
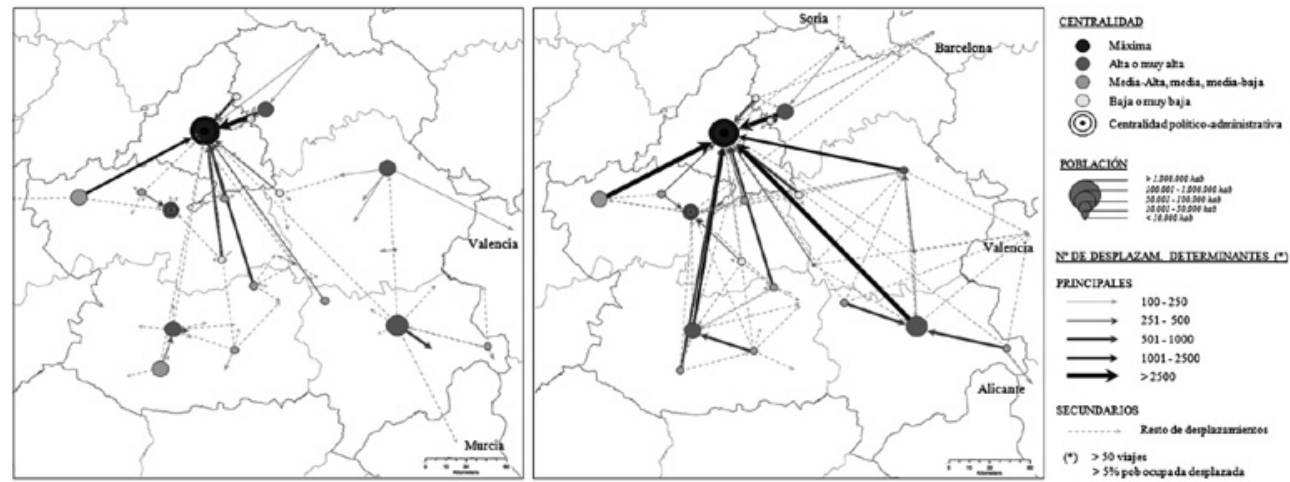

Fuente: Encuesta Propia de 2012

\section{CONCLUSIONES}

El presente artículo complementa los recientes estudios sobre RMM mediante un análisis de sus territorios distantes, tratando de entender si la complejidad de los patrones de movilidad en entornos próximos a la metrópoli (40-50 km, aprox.) igualmente caracteriza los patrones de relaciones funcionales de territorios más alejados (hasta unos $250 \mathrm{~km}$ ).

Por otro lado, cabría señalar que aunque el artículo aborda el caso de estudio de la RMM madrileña, las conclusiones extraídas son útiles para sistemas urbanos de Regiones Metropolitanas que progresivamente amplían sus límites y comienzan a sufrir transformaciones hacia modelos más multicéntricos (aunque la metrópoli sigue ejerciendo un papel principal en la organización del territorio y la atracción de flujos). No obstante, la generalización de las conclusiones aquí extraídas dependerá en cierta medida del tamaño poblacional y la proximidad de las capitales regionales al centro metropolitano.

10 Puesto que los flujos por motivo de negocio son menos frecuentes que los de commuting y un menor número de trabajadores viajan por este motive, con el fin de mostrar una imagen precisa de la situación actual, los límites para identificar los flujos determinantes se han reducido a 50 trabajadores y $5 \%$ de la población desplazada en cada municipio. 
Los resultados empíricos muestran que estas áreas distantes han aumentado de manera notoria sus flujos laborales hacia otros municipios, siendo estos además más complejos. Esta mayor complejidad genera nuevas relaciones, además de las tradicionales hacia la capital provincial: tangenciales entre territorios de estas áreas distantes, radiales hacia la tradicional área metropolitana e interregionales con otras regiones españolas.

Sin embargo, estos nuevos patrones multicéntricos de las relaciones intermunicipales de territorios distantes de RMM están mucho menos desarrollados que los de las áreas más próximas. Es decir, a pesar de que nuevas relaciones horizontales están emergiendo entre municipios distantes de la metrópoli (más significativos para los flujos de negocios), la redistribución de desplazamientos hacia patrones más complejos caracterizando áreas metropolitanas con un nivel de articulación elevado, no son tan evidentes para territorios distantes donde los flujos son principalmente jerárquicos, bien hacia la capital provincial y hacia la metrópoli.

No obstante, diferentes patrones espaciales comienzan a aparecer y pueden ser identificados en estas áreas distantes:

- Municipios próximos a la metrópolis, como son Azuqueca de Henares, El Casar, Ocaña o Tarancón para el caso de Castilla-La Mancha, mantienen o aumentan sus flujos radiales, siendo los únicos municipios con una cierta vinculación con otros subcentros metropolitanos.

- La capacidad de polarización de la metrópolis se traduce en que hasta una determinada distancia (en el caso madrileño, en torno a $150 \mathrm{~km}$ ) los desplazamientos radiales son más intensos que los jerárquicos hacia las capitales provinciales.

- A pesar de que las nuevas capitales regionales consolidadas en estos territorios distantes y las infraestructuras de transporte están facilitando la lenta aparición de nuevas relaciones tangenciales (generando patrones de movilidad más complejos), la mayor parte de los flujos determinantes principales continúan siendo radiales o jerárquicos hacia los tradicionales centros más próximos ${ }^{11}$. La relevancia de los flujos intraprovinciales en áreas distantes pone de manifiesto el débil desarrollo de estructuras policéntricas a nivel intra-regional, donde cada capital provincial continua ejerciendo una influencia significativa, y tan solo un número limitado de viajes (aunque en aumento) tiene lugar con otros territorios más allá de los límites administrativos de cada provincia ${ }^{12}$. Tan solo para el caso de desplazamientos por motivos de negocio, las capacidades de atracción de las nuevas capitales regionales, como es Toledo para el caso de Castilla-La Mancha, pueden ser comparadas con las de los centros metropolitanos, como es Madrid en el caso de estudio, llegando a igualar e incluso superar el total de los radiales. Para los desplazamientos laborales, esta polarización de las capitales regionales no llegan a alcanzar las de la metrópoli (algo que podría deberse al corto período de treinta años transcurrido desde la consolidación de las autonomías españolas y la designación de las capitales regionales).

11 Al igual que Hall y Pain (2006) y de Goei et al. (2010) concluyen para el Sureste de Inglaterra.

12 Estos resultados coinciden con los concluidos por de Goei et al. (2010) y Solís et al. (2012). 
- Aquellos municipios con mayor centralidad (tamaño demográfico, presencia de administración pública, servicios públicos, actividades económicas, etc), como son Albacete, Ciudad Real, Guadalajara o Cuenca en el caso de Castilla-La Mancha, tienden a desarrollar un rol de intermediación, atrayendo un porcentaje importante de flujos desde municipios de centralidad menor y por el contrario, sus flujos intermunicipales comienzan a tener lugar cada vez más con la metrópolis y otros municipios de su misma centralidad.

En definitiva, tres sub-áreas pueden distinguirse en estos territorios distantes de RMM:

- Una primera corona a una cierta distancia de la metrópolis donde las relaciones radiales son de gran intensidad.

- Una zona a una distancia media del centro metropolitano con fuertes relaciones tangenciales, llegando a ser tanto o más importantes que las radiales.

- Un sub-área compuesta por los municipios más distantes con fuertes interacciones hacia otras áreas urbanas periféricas.

Finalmente, en relación a la metodología, el artículo muestra las especificidades de territorios distantes de regiones metropolitanas y confirma que la realización de encuestas a un número determinado de municipios podría ser suficiente para entender sus patrones de movilidad. No obstante, puesto que los resultados apuntan que determinados municipios presentan diferentes relaciones espaciales, las encuestas llevadas a cabo en un futuro deberían prestar mayor atención a estos distintos tipos de municipios. En concreto a los siguientes tres tipos: municipios próximos al tradicional área metropolitana, municipios con alta centralidad y municipios con baja centralidad, tanto en zonas centrales de la región como en zonas más distantes.

\section{BIBLIOGRAFÍA}

AGUILÉRA, A., MIGNOT, D. (2004): «Urban sprawl, polycentrism and commuting. A comparison of seven French urban areas» en Urban Public Economic Review, ${ }^{\circ}$ 1, Vol 1,93-114.

AGUILÉRA, A., MASSOT, M.H. y PROULHAC, L. (2009): «Exploring the relationship between work and travel behaviour on weekdays. An analysis of the Paris Region Travel Survey over 20 years» en Transportation Research Record, $\mathrm{n}^{\circ}$ 2135, 69-77.

BIEHL, D. (1991): «The role of infrastructure in regional development» en Infrastructures and regional development (Vickerman, R.W., ed), London, Edit.Prior, 9-35.

BOARNET, M.G. y SARMIENTO, S. (1998): «Can land-use policy really affect travel behavior? A study of the link between non-work travel and land-use characteristics» en Urban Studies, $\mathrm{n}^{\mathrm{O}} 35$, Vol. 7, 1155-1169.

BURGER, M.J., De GOEI, B., VAN DER LAAN, L. y Huisman, F.J.M. (2011): «Heterogeneous development of metropolitan spatial structure: evidence from commuting patterns in English and Welsh city-regions, 1981-2001» en Cities, n $28,160-170$.

BURNS, M.C., ROCA CLADERA, J., MOIX BERGADÀ, M. y ULIED SEGUÍ, M. (2009) «El sistema metropolitan de la macrorregión de Madrid» en Urban, n ${ }^{\circ}$ 14, 72-79. 
CASTAÑER, M., VICENTE, J. y BOIX, G. (Ed.) (2000): Áreas urbanas y movilidad laboral en España. Girona. Servicio de Publicaciones de la Universidad de Girona.

CLARK, W.A. y KUIJPERS-LINDE, M. (1994): «Commuting in Restructuring Urban Regions» en Urban Studies, n $3,465-483$.

CEBRIÁN, F. y CEBRIÁN, A. (2000): «Los desequilibrios en la estructura urbana de Castilla La Mancha» en Papeles de Geografía, $\mathrm{n}^{\circ}$ 32, 42-59.

CERVERO, R. (1996): «Mixed land-uses and commuting: evidence from the American Housing Survey» en Transportation Research A, $\mathrm{n}^{\circ} 30$, Vol. 5, 361-377.

CERVERO, R. y KOCKELMAN, K. (1997): «Travel demand and the 3Ds: density, diversity, and design» en Transportation Research D, $\mathrm{n}^{\circ} 3,199-219$.

CERVERO, R. y WU K.L. (1998): «Sub-centring and commuting: evidence from the San Francisco bay area» en Urban Studies, n ${ }^{\circ}$ 35, Vol. 7, 1059-1076.

De GOEI, B., BURGER, M.J., VAN OORT, F.G. y KITSON, M. (2010): «Functional polycentrism and urban network development in the Greater South East UK: Evidence from Commuting Patterns, 1981-2001» en Regional Studies, $n^{\circ} 44,1149-1170$.

DGT (2008): Anuario Estadístico General 2008. Madrid. Dirección General de Tráfico

DGT (2011): Anuario Estadístico General 2011. Madrid. Dirección General de Tráfico

FAULCONBRIDGE, J.R., BEAVERSTOCK, J.V., DERUDDER, B. y WITLOX, F. (2009): «Corporate ecologies of business travel in professional service firms. Working towards a research agenda» en EURS, $\mathrm{n}^{\circ}$ 16, Vol. 3, 295-308.

FERIA, J.M. y ALBERTOS, J.M. (Coord.) (2010): La ciudad metropolitana en España: procesos urbanos en los inicios del siglo XXI. Pamplona. Thomson Reuters.

FILION, B., BUNTING, T. y WARRINER, K., (1999): «The entrenchment of urban dispersion: residential preferentes and location patterns in the dispersed city» en Urban Sudies, $n^{\circ} 36$, Vol 8, 1317-1347.

FINDLAY, A.M., STOCKDALE, A., FINDLAY, A. y SHORT, D. (2001): «Mobility as a Driver of Change in Rural Britain: An Analysis of the Links between Migration, Commuting and Travel to Shop Patterns» en International Journal of Population Geography, $\mathrm{n}^{\mathrm{o}} 7,1-15$.

FROST, M., LINNEKER, B. y SPENCE, N. (1998): «Excess or wasteful commuting in a selection of British cities» en Transportation Research A, $\mathrm{n}^{\circ} 32,529-538$.

GALLO, M.T., GARRIDO, R., VIVAR, M. (2010): «Cambios territoriales en la Comunidad de Madrid: policentrismo y dispersión» en EURE, n 36, Vol. 107, 49-65.

GARCÍA-PALOMARES, J.C. (2010): «Urban sprawl and travel to work: the case of the metropolitan area of Madrid» en Journal of Transport Geography, n 18, 197-213.

GARMENDIA, M., UREÑA, J.M. y CORONADO, J.M. (2011): «Cambios en la estructura territorial debidos a nuevas conexiones de alta velocidad en territorios aislados: la provincia de Ciudad Real en España» en EURE, n 37, Vol. 110, 89-115.

GILLI, F. (2005): «Le Bassin parisien. Une région métropolitaine» en Cybergeo: European Journal of Geography 305. (Consultado el 15 de marzo de 2013). http://cybergeo revues.org/3257

GIULIANO, G., SMALL, K.A., (1993): «Is the journey to work explained by urban structure?» en Urban Studies, n 9, 1485-1500.

GIULIANO, G. y GILLESPIE, A. (1997): «Research issues regarding societal change and transport» en Journal of Transport Geography, n ${ }^{\circ}$, Vol. 3, 165-176. 
GIULIANO, G. (1998): «Urban travel patterns» en Modern Transport Geography (Hoyle, B. y Knowles, R., coord.) London, Edit.Wiley, 115-134.

GORDON, P., RICHARDSON, H. y JUN, M.J. (1991): «The commuting paradox: evidence from the top twenty» en Journal of the American Planning Association, $\mathrm{n}^{\circ}$ 57, 416-420.

GUTIÉRREZ-PUEBLA, J. y GARCÍA-PALOMARES, J.C. (2007): «New spatial patterns of mobility within the metropolitan area of Madrid: towards more complex and dispersed flow networks» en Journal of Transport Geography, n 15, Vol. 1, 18-30

HALL, P. y PAIN, K. (2006): The Polycentric Metropolis. London. Earthscan

INE (2011): Encuesta de Población Activa. Tercer trimestre de 2011. Notas de Prensa.

LE NÉCHET, F. (2012): «Urban spatial structure, daily mobility and energy consumption: a study of 34 European cities» en Cybergeo: European Journal of Geography 580 (Consultado el 15 de marzo de 2013). http://cybergeo.revues.org/24966

LEVINSON, D.M. (1998): «Accessibility and the journey to work» en Journal of Transport Geography, ${ }^{\circ}$ 6, Vol. 1, 11-21.

LIMTANAKOOL, N., DIJST, M., y SCHWANEN, T. (2007a): «A Theoretical framework and methodology for characterizing national urban systems on the basis of flows of people: empirical evidence for France and Germany» en Urban Studies, n ${ }^{\circ} 44$, Vol. 11, 2123 2145.

LIMTANAKOOL, N., DIJST, M., y SCHWANEN, T. (2007b): «Ranking functional urban regions: A comparison of interaction and node attribute data» en Cities, $\mathrm{n}^{\circ}$ 24, Vol. 1, 26-42.

LIMTANAKOOL, N., SCHWANEN, T. y DIJST, M., (2009): «Developments in the Dutch Urban System on the Basis of Flows» en Regional Studies, n 43, Vol. 2, 179-196.

MA, K.R. y BANISTER, D. (2006): «Excess Commuting: A Critical Review» en Transport Reviews: A Transnational Transdisciplinary Journal, $\mathrm{n}^{\circ}$ 26, Vol. 6, 749-767.

MARTIN, C. (2000). «La mujer en el mercado de trabajo». Anales de Economía Aplicada. XIV Reunión ASEPELT-España. 22-23 Junio 2000. Oviedo

MCQUAID, R.W. (2009): «A model of the travel to work limits of parents» en Research in Transportation Economics, $\mathrm{n}^{\circ} 25$, Vol. 1, 19-28.

MENDEZ, R. y RODRÍGUEZ MOYA, J. (2007) «Transformaciones productivas y nuevas formas urbanas: difusión de las actividades económicas en la región metropolitana funcional de Madrid». Anales de Geografía, 27 (2), pp. 105-134.

NELSON D. y NILES J. (2000): «Observations on the Causes of Nonwork Travel Growth». 79th Meeting of the Transportation Research Board. January 9-13, 2000. Washington, D.C.

PAZOS OTÓN, M. (2005): Pontevedra Litoral: Hacia una ciudad continua. Pontevedra: Servicio de Publicaciones de la Diputación Provincial de Pontevedra.

PILLET, F., et al. (1980): «Áreas de influencia socioeconómicas de la provincia de Ciudad Real» en Almud, $\mathrm{n}^{\circ}$ 1, 33-65.

PILLET, F., CAÑIZARES, M.C., RUIZ, A.R., MARTÍNEZ, H.S., PLAZA, J.J. y SANTOS, J.F. (2010): «El policentrismo en Castilla-La Mancha y su análisis a partir de la población vinculada y el crecimiento demográfico» en Scripta Nova vol. XIV, núm. 321, 20 de abril de 2010. Universidad de Barcelona. Disponible en http://www.ub.edu/geocrit/sn/sn-321.htm

REQUES, P., DE COS, O. y MARAÑÓN, M. (2012): «Demographic and Socio-economic Context of Spatial Development in Spain». En Territorial Implications of High Speed Rail. A Spanish Perspective (Ureña, J.M. (Ed.), Aldershot: Ashgate, 55-83. 
ROUWENDAL, J. y RIETVELD, P. (1994): «Changes in commuting distances of Dutch households» en Urban Studies, $\mathrm{n}^{\circ}$ 31, Vol. 9, 1545-1557.

SCHWANEN, T., DIELEMAN, F.M. y DIJST, M. (2001): «Travel behavior in Dutch monocentric and polycentric urban systems» en Journal of Transport Geography, $\mathrm{n}^{\circ}$ 9, 173-186.

SICK, T.A. y HARDER, H. (2008): «Exploratory mapping of commuter flows in England and Wales» en Journal of Transport Geography, $\mathrm{n}^{\circ}$ 16, 90-99.

SOLIS, E., UREÑA, J.M. y RUIZ-APILÁNEZ, B. (2012): «Transformación del sistema urbano-territorial en la región central de la España peninsular: la emergencia de la región metropolitana policéntrica madrileña» en Scripta Nova vol. XVI, núm. 420, 20 de noviembre de 2012. Universidad de Barcelona. Disponible en http://www.ub.edu/geocrit/ $\mathrm{sn} / \mathrm{sn}-420 . \mathrm{htm}$

TITHERIDGE, H. y HALL, P. (2006): «Changing travel to work patterns in South East England» en Journal of Transport Geography, $\mathrm{n}^{\circ}$ 14, 60-75.

UREÑA, J.M. y MURUZÁBAL, J.J. (2006): «Sostenibilidad y eficiencia económica en el transporte de la Comunidad de Madrid: evolución de la última década» en Revista del Instituto de Estudios Económicos, $\mathrm{n}^{\circ} 1 \& 2,191-222$.

VALENZUELA RUBIO, M. (2011) «La planificación territorial de la Región Metropolitana de Madrid. Una asignatura pendiente» en Cuadernos Geográficos, 47(2), 95-129.

VAN DER LAAN, N. (1998): «Changing urban systems: an empirical analysis at two spatial levels» en Regional Studies, ${ }^{\circ}$ 32, 235-247.

VAN DE COEVERING, P. y SCHWANEN, T. (2006): «Re-evaluating the impact of urban form on travel patterns in Europe and North America» en Transport Policy, $\mathrm{n}^{\circ} 13,229-239$. 
용

崖

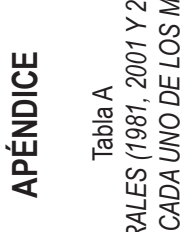
ㄴ.

क

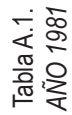

잉

空㟧

留

岂出

종

S

कo

㟔崖

$\gtrless$

ญับ

บ

ํำ

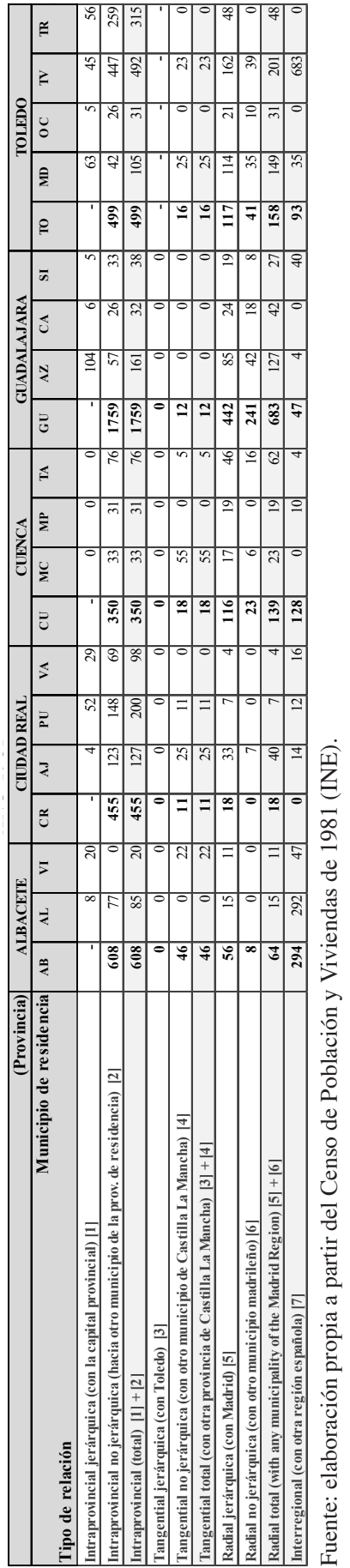

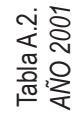

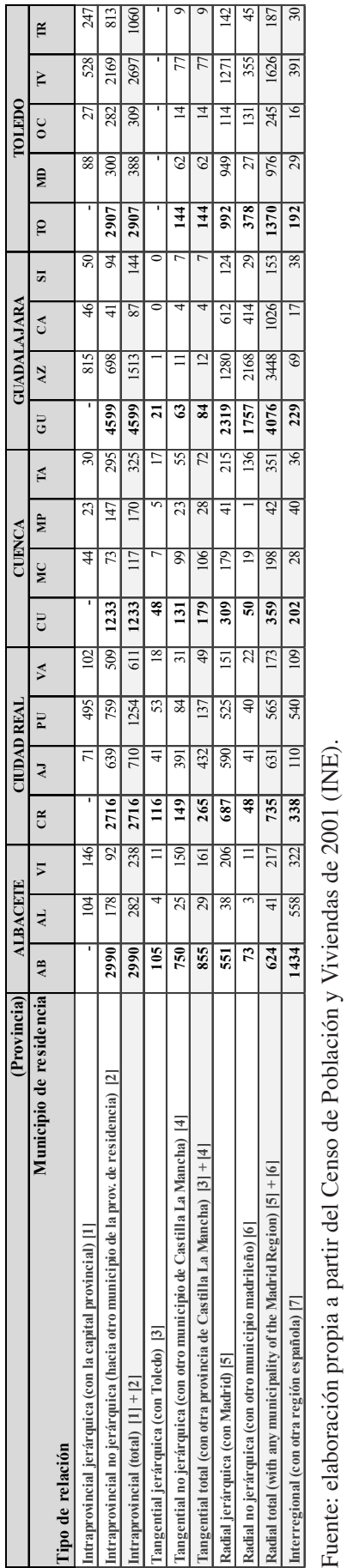




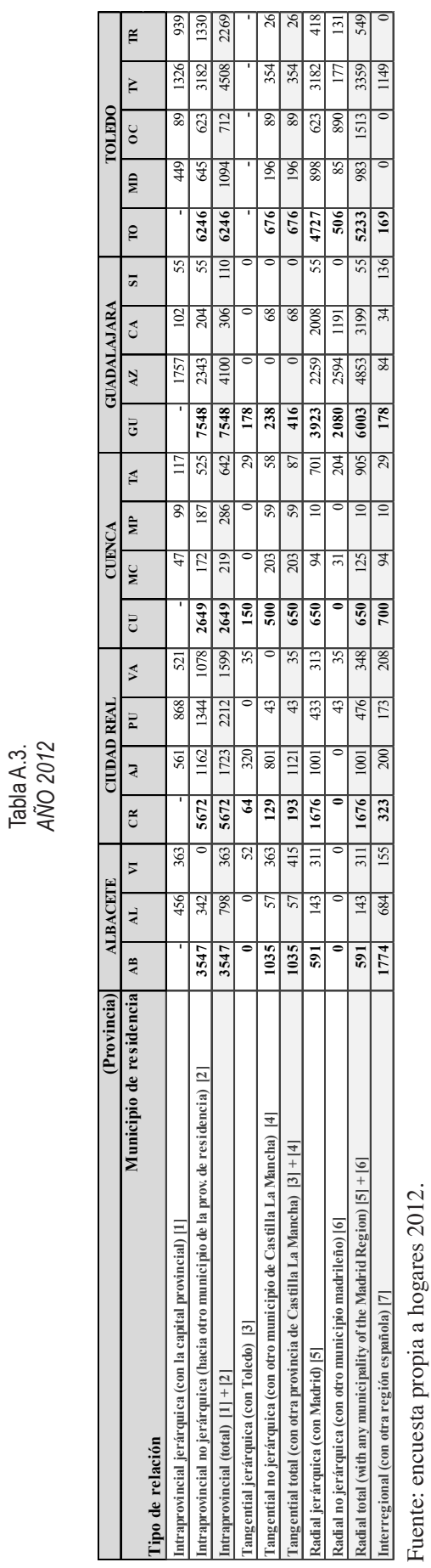

